\title{
Lung Ultrasound in Covid-19: A useful diagnostic tool
}

\author{
Gianmarco Secco, ${ }^{1}$ Marzia Delorenzo, ${ }^{1}$ Caterina Zattera, ${ }^{1}$ Bianca Giacomuzzi Moore, ${ }^{2}$ \\ Lorenzo Demitry, ${ }^{1}$ Giulia Vezzoni, ${ }^{1}$ Flavia Resta, ${ }^{1}$ Bruno Barcella, ${ }^{1}$ Giovanni Cappa, ${ }^{1}$ \\ Tiziano Perrone, ${ }^{3}$ Francesco Salinaro, ${ }^{1}$ Stefano Perlini ${ }^{1}$
}

${ }^{1}$ Emergency Medicine and Emergency Medicine Postgraduate Training Program, IRCCS Policlinico San Matteo Foundation, Department of Internal Medicine University of Pavia, Pavia, Italy; ${ }^{2}$ Internal Medicine, Hôpital de Morges (Ensemble Hopitalier de la Côte), Morge, Switzerland; ${ }^{3}$ Internal Medicine, IRCCS Policlinico San Matteo Foundation, Department of Internal Medicine University of Pavia, Pavia, Italy

\begin{abstract}
Coronavirus disease 2019 (Covid-19), caused by a novel enveloped RNA betacoronavirus, has recently been declared a public health emergency by the World Health Organization (WHO). The lack of knowledge at the beginning of the pandemics, associated with the inherent risk of infective spreading, makes initial recognition and management particularly complex, in terms of defining effective diagnostic and therapeutic protocols. In the Emergency setting, Lung Ultrasound (LUS) can play an important role in the management of patients with SARS-CoV2-related pneumonia, expanding from the initial diagnosis to the subsequent monitoring and follow-up. Among many other potential advantages (such has the absence of ionizing radiation, its inherent costeffectiveness, and bedside repeatability), LUS provides immediate diagnostic response and might prevent the risk of spreading the infection by moving the patient from the Emergency Room to the Radiology facilities. Aim of this short review is to define the potential role of lung ultrasound in Covid-19 patients, according to the evidence in the medical literature.
\end{abstract}

\section{Introduction}

Coronavirus disease 2019 (Covid-19) is a mainly respiratory infectious disease caused by a novel enveloped RNA betacoronavirus, that has currently been named severe acute respiratory syndrome coronavirus 2 (SARS-CoV-2). As declared by the World Health Organization (WHO), Covid-19 is a public health emergency, reaching a pandemic diffusion. ${ }^{1}$

The first cases of Covid-19 have been detected in December 2019 in Wuhan, China. Since then, the infection has rapidly spread. In Italy, the first case of secondary transmission of Covid19 was confirmed on February 21, 2020 in Codogno (Lodi) in a patient transferred to the San Matteo Hospital in Pavia. The last update on Covid-19 by the Italian Ministry of Health (Apr $6^{\text {th }}$, 2020) reported 132.547 confirmed cases in Italy, with 16.523 deaths. ${ }^{2}$

The clinical manifestations of Covid-19 can vary from a mild self-limiting flu-like syndrome to an interstitial pneumonia that can evolve into Acute Respiratory Distress Syndrome (ARDS), multiorgan failure and potentially death. ${ }^{3}$ As a disease that predominantly affects the respiratory tract, Lung Ultrasound (LUS) could reasonably play a role in the recognition and management of patients with SARS-Cov2-related pneumonia, from the initial diagnosis to the subsequent follow-up. In the emergency setting, LUS has shown to have greater diagnostic accuracy in differentiating the underlying causes of acute dyspnoea when compared to other traditional methods. . $^{4-6}$

Several major advantages of LUS over other radiological techniques can be identified. In general, LUS is a bedside technique, applicable also to unstable patients, ionizing radiation free, cheap, fast, and easy to repeat. Moreover, it can provide an immediate diagnostic response, and is often performed by the Emergency Medicine Doctor as an invaluable complement to clinical assessment. ${ }^{7}$ The learning curve is steep, and therefore it can be applied in different clinical settings and by different specialists.

During the 2009 Influenza A (H1N1) epidemic, LUS was tested and proposed as a tool to be integrated in the clinical evaluation, useful for differential diagnosis of bacterial pneumonia, especially when chest X-ray does not result diagnostic, as in the early phase of the disease. ${ }^{8}$

\section{Basis for lung ultrasound}

LUS has significantly evolved over the last years and it is widely used in the emergency setting for the management of critically ill patients. In general, LUS provides a lung representation based both on images and artefacts. Through the alterations affect- 
ing the ratio between air and fluid in the superficial lung, LUS allows to define the distinction between solid and fluid lesions and to identify the alveolar-interstitial syndrome. ${ }^{9}$ Care should be taken in the probe choice, machine setting and acquisition protocols, as they could influence the interpretation of the LUS images. LUS must be performed according to a systematic protocol of examination. Although different probes might be suitable for LUS, convex and linear probes are most widely used. The linear probe (9-12 $\mathrm{MHz}$ ) has high superficial definition and low penetration capacity due to its high frequency. It is therefore useful in the evaluation of superficial structures, especially the pleural line. The convex probe (2.5-5 MHz) has higher capacity of penetration, being ideal for the study of the parenchymal structure, although less precise in the evaluation of more superficial structures such as the pleura.

Specific settings of the machine are required to obtain a correct execution of LUS: removal of harmonic-imaging and lowering of the total gain (which otherwise would diminish the presence of artefacts); elimination of multi-focus modality and focus setting at the level of the pleural line; depth setting at $6-10 \mathrm{~cm}$ from the pleural line (for full appreciation of the extension of B-lines). ${ }^{10}$ Different LUS acquisition protocols have been validated so far. ${ }^{11}$ One of these, that is frequently used in emergency setting, consists in performing LUS acquisition of twelve areas, after systematic scan of each hemithorax in six regions: two anterior, two lateral and two posterior regions, according to anatomical landmarks set by anterior and posterior axillary lines (Figure 1). ${ }^{12}$

When performing LUS we can find different scenarios with a progressive evolution of the presence of artefacts concomitant with the increase of the lung 'liquid' component. The 'normal lung' pattern is characterized by A lines, reverberation of horizontal artefacts parallel to the pleural line, which are repetitive at a constant distance equal to the distance between the pleural line and the probe surface. Lung aeration approaches 100\%. As the air/fluid ratio decreases, B lines artefacts start to appear. B-lines are vertical laser-like, hyperechoic image, arising from the pleural line, extending to the bottom of the screen without significant fading, and synchronously moving with lung-pleural sliding. When the lung aeration decreases, the number of vertical artefacts progressively increase, until they become more and more coalescent up to the 'white lung sign'. When the air content of the lung is approaching $0 \%$, the acoustic pattern is such that the lung appears similar to an intra-abdominal parenchymal organ, that is defined as "tissuelike"13 (Figure 2). Since LUS provides a regional representation of the different lung areas, it is possible to make a qualitative estimation of the extent of aeration loss, regardless the etiology. An ultra- sound grading system, that is mainly used in the Intensive Care Unit (ICU) setting, identifies four patterns of progressive loss of aeration, providing the following scores:

- Pattern 0 (score 0): A lines on the whole field or up to 2 separate $\mathrm{B}$ lines.

- Pattern 1 (score 1): Three or more well-spaced B lines.

- Pattern 2 (score 2): Coalescent B lines.

- Pattern 3 (score 3): "Tissue-like pattern".

The sum of the regional scores represents the LUS SCORE, ranging from 0 (well-aerated lung) to 36 (all regions are consolidated). This score represents a useful tool to monitor the lung status, and it has been shown that in patients with ARDS regional LUS score is strongly correlated with the tissue density as assessed by $\mathrm{CT}$ scan. ${ }^{14}$

Although interpretation of ultrasound vertical artifacts repre-

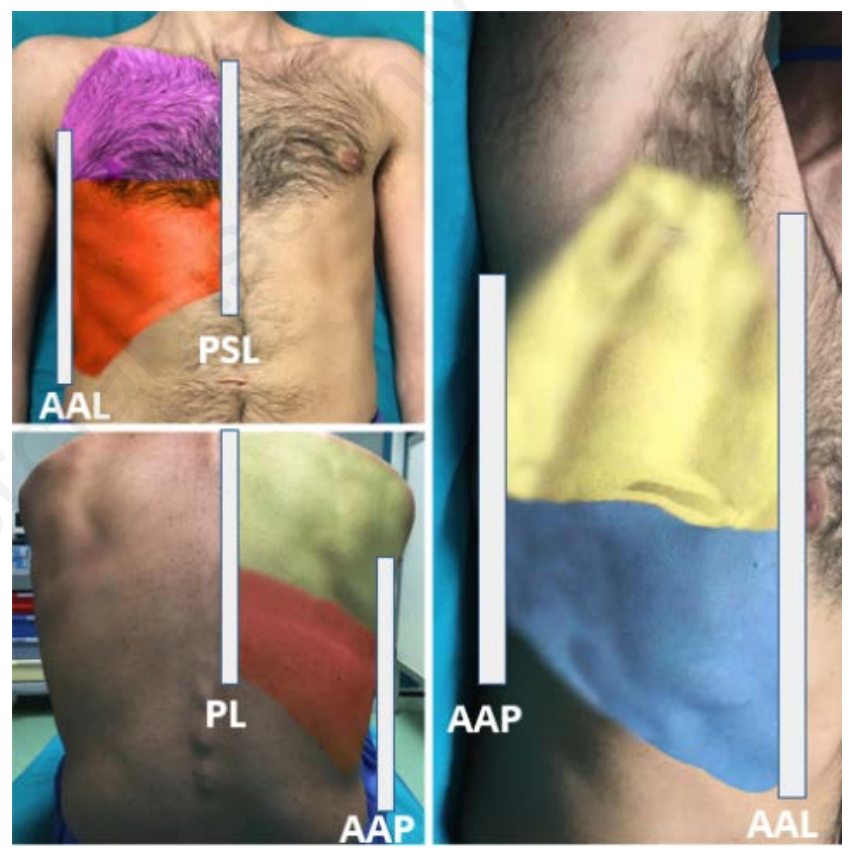

Figure 1. Hemithorax division. PSL: parasternal line; AAL: anterior axillary lines; AAP: posterior axillary lines; PL: paravertebral line.

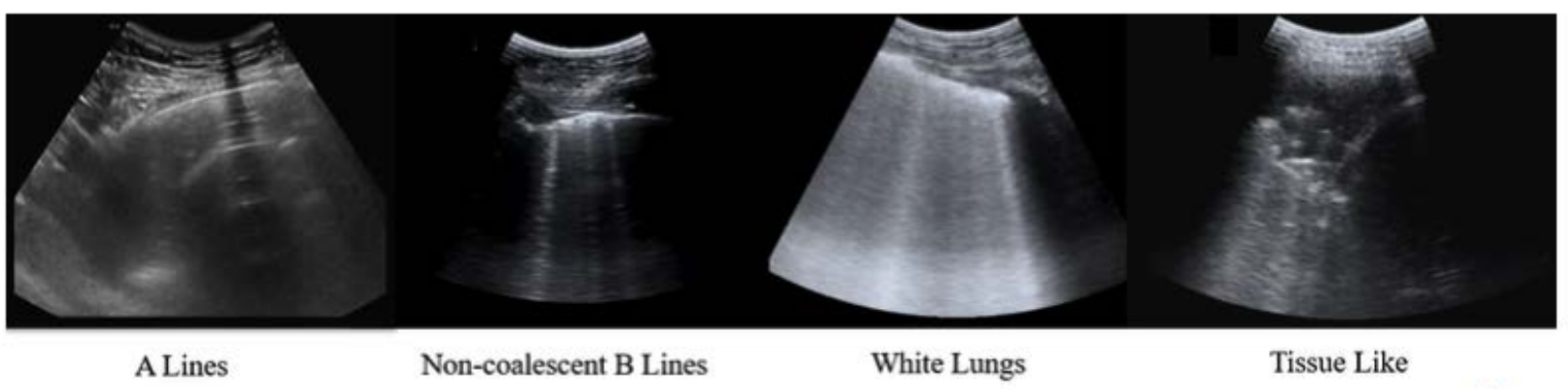


sents the differential diagnosis element standing between a normally aerated lung and a lung with an interstitial disease, many studies considered only the number of vertical artifacts as a sign of severity. Multiple ultrasound signal reflections, when trapped inside the channels created by interaction between the air spaces, when a pathologic condition afflicts the lung, create vertical artifacts. ${ }^{15,16}$ Different lung diseases in different stages determine different acoustic traps in terms of configuration, volume and shape, therefore vertical artifacts look different. Recent observations suggest that the description of the artefacts in term of variability and internal structure could have an important role in the characterization of the lung surface, directing the diagnosis, and identifying different groups of lung diseases. Despite its extensive clinical use, up to now 'B-lines' is a term that simplifies a wide variety of acoustic interactions over the pleural plane, because many mechanisms and lung configurations are able to generate these artifacts. ${ }^{16}$

\section{Bedside lung ultrasound in Covid-19 patients}

Chest computerized tomography (CT) plays an important role in screening suspected Covid-19 patients, since it may identify lung lesions before clinical manifestation and nucleic acid detection. ${ }^{17,} 18$

The use of traditional imaging such as Chest X-Ray or CT scan, in most centre, require patient's transfer to the radiology unit with the potential risk of exposure to the high contagiousness of Covid-19. In different settings, LUS has been shown to be superior (or at least not inferior) to standard Chest X-Ray in the Emergency Room evaluation of pneumonia and/or adult respiratory distress syndrome (ARDS), with some extra advantages such as bedside use, repeatability, radiation-free, and low cost. ${ }^{19}$

Some preliminary data show that LUS features of Covid-19 pneumonia/ARDS are related to disease's stage and to lung injury severity. In a recent letter, Peng et al. ${ }^{20}$ observed that in 20 Covid19 patients LUS finding range from mild alveolar interstitial pattern, to severe bilateral interstitial pattern, to lung consolidation. Soldati et al. ${ }^{21}$ have considered the LUS patterns of subjects with Covid-19 pneumonia as quite characteristic. They described that the first pulmonary manifestations are represented by a patchy distribution of interstitial artefactual signs (single and/or confluent vertical artefacts, small white lung regions). Subsequently, these vertical artefacts extend to multiple areas of the lung surface. Another aspect is represented is represented by the presence, still patchy, of small subpleural consolidation with associated areas of white lung (Figure 3). Lung lesions can evolve in consolidation, especially in a gravitational position, with or without dynamic air bronchograms, increasing their extension and being related to a progressive worsening of respiratory insufficiency, that may eventually need invasive ventilatory support. The advanced Covid-19

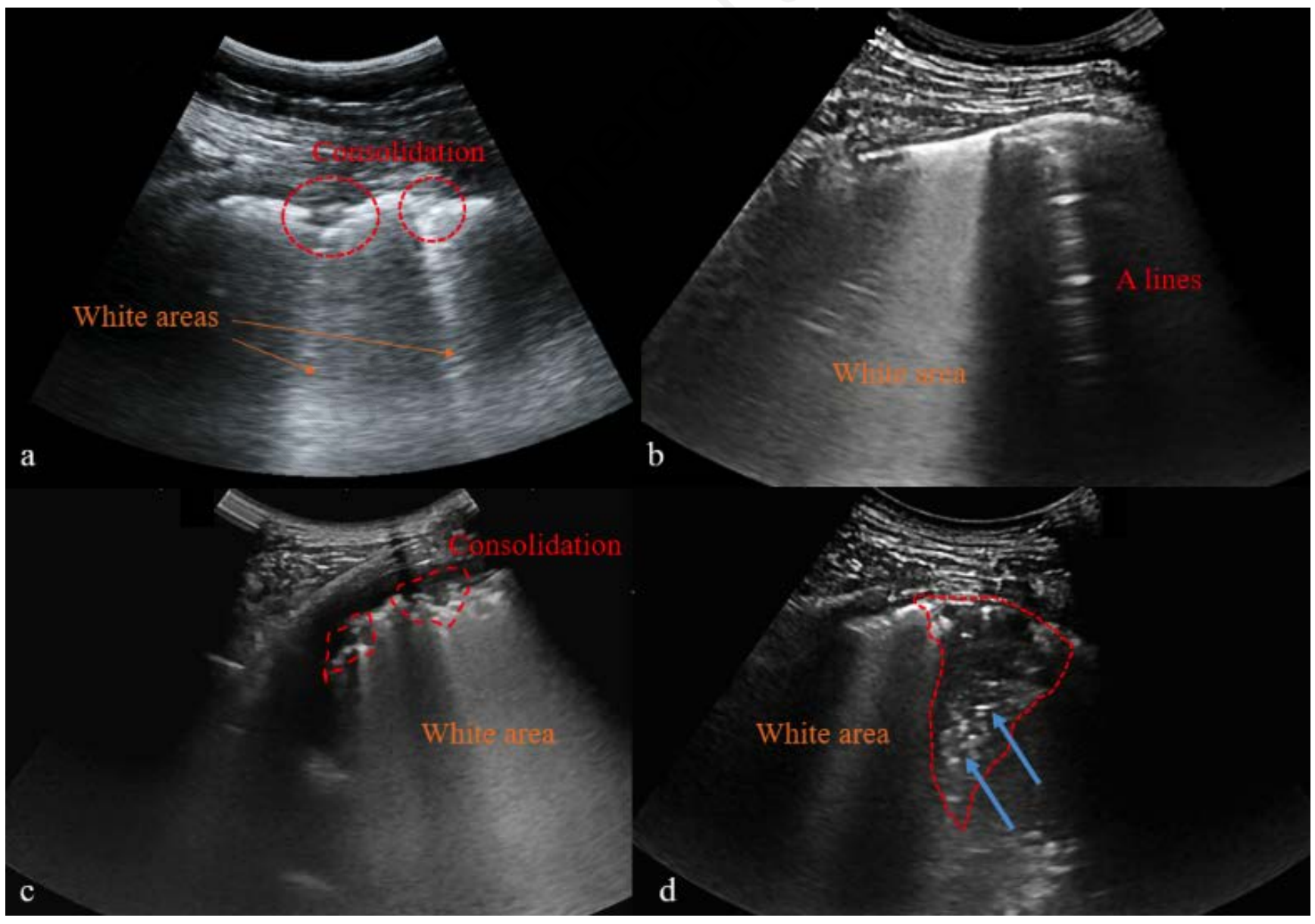

Figure 3. Pulmonary manifestations of Covid-19. a-c) Small subpleural consolidation with associated areas of white lung; b) Small white lung regions and normal lung pattern; d) Consolidation with associated dynamic air bronchograms (blue arrows). 
pneumonia features are similar to ARDS, and show evident consolidation, especially in the postero-basal regions, with widespread and irregular vertical artefactual changes. ${ }^{21}$

In a recent communication by Huang et al., ${ }^{22}$ the ultrasonic manifestations of 20 peripulmonary lesions of non-critical Covid19 are in agreement with Soldati et al. ${ }^{21}$ The lesions are mostly located in the posterior fields of both lungs, with continuous or discontinuous or coalescent (White lung sign) vertical artefacts. Compared with the B lines caused by cardiogenic pulmonary oede$\mathrm{ma}$, the $\mathrm{B}$ lines here were more likely to be fused and fixed and irregular ${ }^{14}$.

In summary, the Covid-19 foci are mainly observed in the posterior fields bilaterally, especially the

posterior and inferior fields. Vertical artefacts visible are single and/or fused, with small white lung regions. The pleural line is discontinuous or interrupted with spared areas. The subpleural lesions appear patchy, in

which air bronchogram sign can be seen and can be associated with areas of withe lung. In the Supplementary data link, we here report a quick guide to LUS in this setting.

\section{Conclusions}

The similarities in other pathologies, the current clinical evidence, the theoretical bases of LUS and its widespread use in different clinical setting suggest a potential diagnostic accuracy of LUS. Application of LUS has allowed to identify patients with lung involvement and to stage their severity. The possibility of performing LUS bedside minimizes the potential risk of infection spreading within the healthcare personnel. In conclusion, recent literature evidence in Covid-19 patients together with the current practical experience endorse LUS as a useful diagnostic tool for the fight against the current pandemic disease.

\section{References}

1. WHO, 2020. Coronavirus. Available from: https://www. who.int/health-topics/coronavirus\#tab=tab_1

2. Ministero della Salute. [Nuovo Coronavirus. Cosa c'è da sapere]. Available from: http://www.salute.gov.it. 2020. [Italian website].

3. Guan WJ, Ni ZY, Hu Y, et al. Clinical characteristics of Coronavirus disease 2019 in China. The New Engl J Med 2020.doi:10.1056/NEJMoa2002032.

4. Volpicelli G, Elbarbary M, Blaivas M, et al. International evidence-based recommendations for point-of-care lung ultrasound. Intensive Care Med 2012;38:577-91. doi: 10.1007/ s00134-012-2513-4.

5. Perrone T, Maggi A, Sgarlata C, et al. Lung ultrasound in internal medicine: a bedside help to increase accuracy in the diagnosis of dyspnea. Eur J Intern Med 2017;46:61-5. doi: 10.1016/j.ejim.2017.07.034.

6. Cortellaro F, Colombo S, Coen D, Duca PG. Lung ultrasound is an accurate diagnostic tool for the diagnosis of pneumonia in the emergency department. Emerg Med J 2012;29:19-23. doi: 10.1136/emj.2010.101584.

7. Lichtenstein DA. Ultrasound in the management of thoracic disease. Crit Care Med 2007;35:S250-61.

8. Testa A, Soldati G, Copetti R, et al. Early recognition of the 2009 pandemic influenza A (H1N1) pneumonia by chest ultrasound. Crit Care 2012;16:R30. doi: 10.1186/cc11201.

9. Lichtenstein D, Meziere G, Biderman P, et al. The comet-tail artifact. An ultrasound sign of alveolar-interstitial syndrome. Am J Respir Crit Care Med 1997;156:1640-6.

10. Via G, Storti E, Gulati G, et al. Lung ultrasound in the ICU: from diagnostic instrument to respiratory monitoring tool. Minerva Anestesiol 2012;78:1282-96.

11. Volpicelli G, Elbarbary M, Blaivas M, et al. International evidence-based recommendations for point-of-care lung ultrasound. Intensive Care Med 2012;38:577-91. doi: 10.1007 /s00134-012-2513-4.

12. Bouhemad B, Mongodi S, Via G, Rouquette I. Ultrasound for "lung monitoring" of ventilated patients. Anesthesiol 2015; 122:437-47.

13. Soldati G, Copetti R, Gargani L. [Lung sonography for the cardiologist]. G Ital Cardiol (Rome). 2007;8:139-47. [Article in Italian].

14. Mojoli F, Bouhemad B, Mongodi S, Lichtenstein D. Lung Ultrasound for Critically Ill Patients. Am J Respir Crit Care Med 2019;199:701-14. doi: 10.1164/rccm.201802-0236CI.

15. Demi M, Prediletto R, Soldati G and Demi L. Physical Mechanisms Providing Clinical Information From Ultrasound Lung Images: Hypotheses and Early Confirmations. IEEE Trans Ultrason Ferroelectr Freq Control 2020;67:612-23. doi: 10.1109/TUFFC.2019.2949597.

16. Soldati G, Demi M, Inchingolo R, et al. On the Physical Basis of Pulmonary Sonographic Interstitial Syndrome. J Ultrasound Med 2016;35:2075-86. doi: 10.7863/ultra.15.08023.

17. Ai T, Yang Z, Hou H, et al. Correlation of Chest CT and RTPCR Testing in Coronavirus Disease 2019 (COVID-19) in China: A Report of 1014 Cases. Radiol 2020:200642. doi: https://doi.org/10.1148/radiol.2020200642.

18. Bernheim A, Mei X, Huang M, et al. Chest CT Findings in Coronavirus Disease-19 (COVID-19): Relationship to Duration of Infection. Radiol 2020:200463. doi: doi: 10.1148 /radiol.2020200463.

19. Mayo PH, Copetti R, Feller-Kopman D, et al. Thoracic ultrasonography: a narrative review. Intensive Care Med 2019;45:1200-11. doi: 10.1007/s00134-019-05725-8.

20. Peng QY, Wang XT, Zhang LN, et al. Findings of lung ultrasonography of novel corona virus pneumonia during the 20192020 epidemic. Intensive Care Med 2020. doi: https://doi. org/10.1007/s00134-020-05996-6.

21. Soldati G, Smargiassi A, Inchingolo R, et al. Is there a role for lung ultrasound during the Covid-19 pandemic? J Ultrasound Med 2020. doi https://doi.org/10.1002/jum.15284.

22. Huang Y, Wang S, Liu Y, et al. A preliminary study on the ultrasonic manifestations of peripulmonary lesions of non-critical novel Coronavirus pneumonia (Covid-19). SSRN 2020. 